\title{
Origin of the periodic structure in the conductance curve of gold nanojunctions in hydrogen environment
}

\author{
Zhuoling Jiang, ${ }^{1}$ Hao Wang, ${ }^{1}$ Stefano Sanvito, ${ }^{2}$ and Shimin Hou ${ }^{1,3, *}$ \\ ${ }^{1}$ Centre for Nanoscale Science and Technology, Key Laboratory for the Physics and Chemistry of Nanodevices, Department of Electronics, \\ Peking University, Beijing 100871, China \\ ${ }^{2}$ School of Physics, AMBER and CRANN Institute, Trinity College, Dublin 2, Ireland \\ ${ }^{3}$ Beida Information Research (BIR), Tianjin 300457, China
}

(Received 4 January 2016; published 31 March 2016)

\begin{abstract}
The evolution of the atomic structure and the vibrational and electronic transport properties of gold atomic junctions incorporating molecular and atomic hydrogen upon elongation have been investigated with the nonequilibrium Green's function formalism combined with density functional theory. Our calculations show that for the case of gold junctions doped with a single $\mathrm{H}_{2}$ molecule the low-bias conductance drops rapidly with the electrodes' separation, while it remains almost constant if a single $\mathrm{H}$ atom replaces the molecule. In contrast, when one considers two $\mathrm{H}$ atoms adsorbed on a gold monatomic chain forming an $\mathrm{Au}-\mathrm{H}-\mathrm{Au}-\mathrm{H}-\mathrm{Au}$ double-bridge structure, the low-bias conductance increases first and then shows a plateau upon stretching the junction, in perfect agreement with experiments on gold nanocontacts in hydrogen environment. Furthermore, also the distribution of the calculated vibrational energies of the two $\mathrm{H}$ atoms is consistent with the experimental result in the low-conductance region, demonstrating clear evidence that hydrogen molecules can dissociate on stretched gold monatomic chains. These findings are helpful to improve our understanding of the structure-property relation of gold nanocontacts and also provide a new prospect for gold nanowires being used as chemical sensors and catalysts.
\end{abstract}

DOI: 10.1103/PhysRevB.93.125438

\section{INTRODUCTION}

The fabrication of atomic-size gold wires is a milestone in nanotechnology because of the prospects open for the realization of future nanoelectronic devices. The reduced dimensionality of such gold nanowires makes them show unusual mechanical, chemical, and transport properties such as quantized conductance and exceptional reactivity compared to bulk gold [1-3]. In particular, investigations on the effects of molecular and atomic hydrogen on their atomic structures and electronic transport properties have received extensive attention [4-25]. On the one hand, the presence of hydrogen impurities intercalated between the gold atoms is assumed to explain the striking structure stability of gold nanowires at the large $\mathrm{Au}-\mathrm{Au}$ separations of 3.6-4.0 $\mathrm{A}$, which have been observed in numerous experiments using transmission electron microscopy and high-resolution transmission electron microscopy [26-30]. This conjecture is based on the fact that hydrogen is present even in high vacuum conditions and cannot be visualized by electron microscopy techniques due to the low contrast in comparison with the heavier gold atoms. Density functional theory (DFT) calculations and ab initio molecular dynamics simulations supported the possibility that the inserted hydrogen impurities would increase the $\mathrm{Au}-\mathrm{Au}$ distance and enhance the structure stability of the nanowires [4-11]. On the other hand, dramatic modifications of the electronic transport properties of gold nanowires have been observed in hydrogen environment. For example, Csonka et al. not only obtained a new peak near $0.5 G_{0}\left(G_{0}=2 e^{2} / h\right.$ is the conductance quantum) superimposed to the low-conductance tail in the conductance histogram of gold nanowires when

\footnotetext{
*Corresponding author: smhou@pku.edu.cn
}

hydrogen molecules were intentionally introduced into the experimental setup [13], but they also found a unique feature, namely, some conductance traces presented a well-defined periodic structure with a pronounced positive slope upon stretching in the low-conductance region of $0.05-0.3 G_{0}$ [14]. At the same time Kiguchi and co-workers studied the influence of hydrogen molecules on $\mathrm{Au}$ atomic contacts using action and inelastic electron tunneling spectroscopy, and found the signature of vibration modes related to bonding between $\mathrm{Au}$ and $\mathrm{H}$ [15]. To be more specific, the majority of the observed vibration modes were located in the $20-55 \mathrm{meV}$ range for junctions having conductance between $0.05 G_{0}$ and $0.5 G_{0}$ and a broad feature was observed at around $33 \mathrm{meV}$ in the vibration energy distribution. Besides these experimental studies a substantial amount of theoretical investigations focused on the mechanical, vibrational, and electronic transport properties of gold nanowires doped with atomic and molecular hydrogen [18-25]. Although previous studies have revealed the role of atomic hydrogen in the origin of the conductance peak near $0.5 G_{0}$ [19], there is still no reasonable explanation for the appearance of the peculiar periodic structure in the conductance curve and the distribution of vibration energies in the low- conductance region [14,15].

In order to address these issues, here we investigate the vibrational and electronic transport properties of gold atomic contacts incorporating molecular and atomic hydrogen by employing the nonequilibrium Green's function formalism combined with density functional theory (that is, the socalled NEGF+DFT approach) [31-40]. Our calculations show that, as the electrode separation is increased, the low-bias conductance of gold junctions incorporating one $\mathrm{H}_{2}$ molecule drops rapidly while the low-bias conductance of gold junctions doped with a single $\mathrm{H}$ atom remains almost constant. In contrast, gold atomic contacts including two $\mathrm{H}$ atoms adsorbed 
at two consecutive bridge sites of a gold monatomic chain, thus forming an $\mathrm{Au}-\mathrm{H}-\mathrm{Au}-\mathrm{H}-\mathrm{Au}$ double-bridge structure, presents an increase of the low-bias conductance in the initial stretching process and then a conductance plateau. This peculiar conductance behavior occurs in junctions with both (100)and (111)-oriented gold electrodes and monatomic chains with different number of gold atoms included. Moreover, the calculated vibrational energies are also distributed in the 20$55 \mathrm{meV}$ range. Thus, gold junctions with an $\mathrm{Au}-\mathrm{H}-\mathrm{Au}-\mathrm{H}-\mathrm{Au}$ double-bridge structure are appropriate junction models to explain both the periodic behavior with positive slope in the conductance curve and the distribution of vibration energies in the low-conductance region observed for gold nanocontacts immersed in a hydrogen environment.

\section{CALCULATION METHOD}

In this work we use the SIESTA code to compute the atomic structure of the Au-H systems and the quantum transport code SMEAGOL to study their electronic transport properties [38-41]. SIESTA is an efficient DFT package, in which the wave functions of the valence electrons are expanded with a finite-range numerical orbital basis set and the atomic cores are described by improved Troullier-Martins pseudopotentials [41,42]. We construct a double-zeta plus polarization basis set for both $\mathrm{Au}$ and $\mathrm{H}$. The generalized gradient approximation within the Perdew-Burke-Ernzerhof formulation is used for the exchange-correlation functional [43]. With our choice of pseudopotentials and basis functions a lattice constant of $4.18 \AA$ is obtained for bulk gold, which is in good agreement with the experimental value of $4.08 \AA$ [44], while the $\mathrm{H}-\mathrm{H}$ bond length and the stretching frequency of the $\mathrm{H}_{2}$ molecule in the gas phase are respectively calculated to be $0.75 \AA$ and $4411 \mathrm{~cm}^{-1}$, consistent with the experimental values of $0.74 \AA$ and $4401 \mathrm{~cm}^{-1}[45,46]$. In the case of the Au-H systems the atomic coordinates in the device region are relaxed until the atomic forces are smaller than $0.01 \mathrm{eV}^{-1}$, and a cutoff of $800 \mathrm{Ry}$ for the real-space grid integration is employed to reduce the so-called egg-box effect [41].

SMEAGOL is a practical implementation of the NEGF+DFT approach, which employs SIESTA as the DFT platform [38-40]. The unit cell of the extended molecule comprises the $\mathrm{H}$ atoms, some $\mathrm{Au}$ atoms with low coordination, and $11 \mathrm{Au}(100)$ or ten $\mathrm{Au}(111)$ atomic layers with a $(3 \times 3)$ in-plane supercell. We always consider periodic boundary conditions in the plane transverse to the transport. The total transmission coefficient $T(E)$ of the junction is evaluated as

$$
T(E)=\frac{1}{\Omega_{2 \mathrm{DBZ}}} \int_{\text {2DBZ }} T(\vec{k} ; E) d \vec{k},
$$

where $\Omega_{2 \mathrm{DBZ}}$ is the area of the two-dimensional Brillouin zone (2DBZ) in the transverse directions. The $k$-dependent transmission coefficient $T(\vec{k} ; E)$ is obtained as

$$
T(\vec{k} ; E)=\operatorname{Tr}\left[\Gamma_{L} G_{M}^{R} \Gamma_{R} G_{M}^{R+}\right],
$$

where $G_{M}^{R}$ is the retarded Green's function matrix of the extended molecule and $\Gamma_{L(R)}$ is the broadening function matrix describing the interaction of the extended molecule with the left (right) electrode. Here, we calculate the transmission coefficient by sampling $3 \times 3 k$ points in the transverse $2 \mathrm{DBZ}$.

\section{RESULTS AND DISCUSSION}

Since Csonka et al. attributed the observed periodic structure in the conductance trace to the pulling of gold monatomic chains incorporating a hydrogen molecule [14], we start our studies by investigating the effects of molecular hydrogen absorption, paying particular attention to the modifications of the low-bias conductance and of the vibrational frequencies at different stretching states of the wire. Figure 1(a) shows a symmetric model of the $\mathrm{Au}-\mathrm{H}_{2}-\mathrm{Au}$ junction, in which one hydrogen molecule is incorporated in the center of a four-atom-long gold monatomic chain connected to two (100)oriented gold electrodes through one pyramidal base at each side. The electrode separation $L$ is the distance between the outermost surface layers of the two gold electrodes, and in our calculations we elongate the junction through small increments of $\Delta L=0.2 \AA$. At each step we relax the positions of the two $\mathrm{H}$ atoms and of the gold atoms both in the wire and in the pyramidal bases, while we keep the gold atoms belonging to the electrodes fixed. The transmission coefficient at the Fermi energy, $E_{\mathrm{F}}$, and some typical bond lengths in the junction as a function of the electrode separation $L$ are respectively shown in Figs. 1(b) and 1(c). As we can see, the presence of the $\mathrm{H}_{2}$ molecule reduces the conductance of the gold monatomic chain from the perfect transmission of a single channel to a partial transmitted regime with a conductance value of $0.74 G_{0}$ at $L=17.82 \AA$. Increasing the electrode separation leads to a dramatic monotonic drop in the junction conductance [20]. Concomitantly, the bond length of the hydrogen molecule $d_{\mathrm{H} 1-\mathrm{H} 2}$ contracts from $0.87 \AA$ at $L=17.82 \AA$ to $0.77 \AA$ at $L=18.62 \AA$, whereas the distance $d_{\text {Au2-H1 }}$ between the $\mathrm{Au}$ atom adjacent to $\mathrm{H}(\mathrm{Au} 2)$ and the $\mathrm{H}(\mathrm{H} 1)$ atom increases from $1.87 \AA$ to $2.32 \AA$. Since the bond length $d_{\mathrm{Au} 1-\mathrm{Au} 2}$ between the Au1 atom and the one next to it (the Au2 atom) remains constant at $2.65 \AA$, we find that the coupling between the $\mathrm{H}_{2}$ molecule and the gold nanowire is continuously weakened following the elongation of the $\mathrm{Au}-\mathrm{H}_{2}-\mathrm{Au}$ junction. This is also the precise reason for the continuous reduction of the low-bias junction conductance, which is dominated by the electronic coupling between the $\mathrm{H} 1 s$ and the Au $6 s$ atomic orbitals. Because $d_{\mathrm{H} 1-\mathrm{H} 2}$ stays constantly at $0.77 \AA$ (noting that the $\mathrm{H}-\mathrm{H}$ bond length in an isolated hydrogen molecule is $0.75 \AA$ ) and $d_{\mathrm{Au} 2-\mathrm{H} 1}$ still increases when further increasing the electrode separation, we can deduce that the $\mathrm{Au}-\mathrm{H}_{2}-\mathrm{Au}$ junction breaks at the $\mathrm{Au}-\mathrm{H}$ interface [24] and the interaction between the $\mathrm{H}_{2}$ molecule and the gold nanowire is not strong enough to pull out a monatomic gold chain from the gold electrode. As such, this structure cannot be responsible for the observation of the periodic behavior of the conductance curve upon stretching. It should be noted that due to its symmetric atomic arrangement, the $\mathrm{Au}-\mathrm{H}_{2}-\mathrm{Au}$ junction shown in Fig. 1(a) can break at either the Au2-H1 bond or the Au3-H2 one depending on the details of the relaxation process.

In addition to the electronic transport properties, we have also analyzed the dependence of the vibration spectrum of the $\mathrm{Au}-\mathrm{H}_{2}-\mathrm{Au}$ junction on the electrode separation [Fig. 1(d)]. The dynamical properties of the $\mathrm{H}_{2}$ molecule can be characterized by four types of vibration modes: the longitudinal stretching mode, the longitudinal translation mode in which the center of mass of the $\mathrm{H}_{2}$ molecule vibrates along the transport direction, 

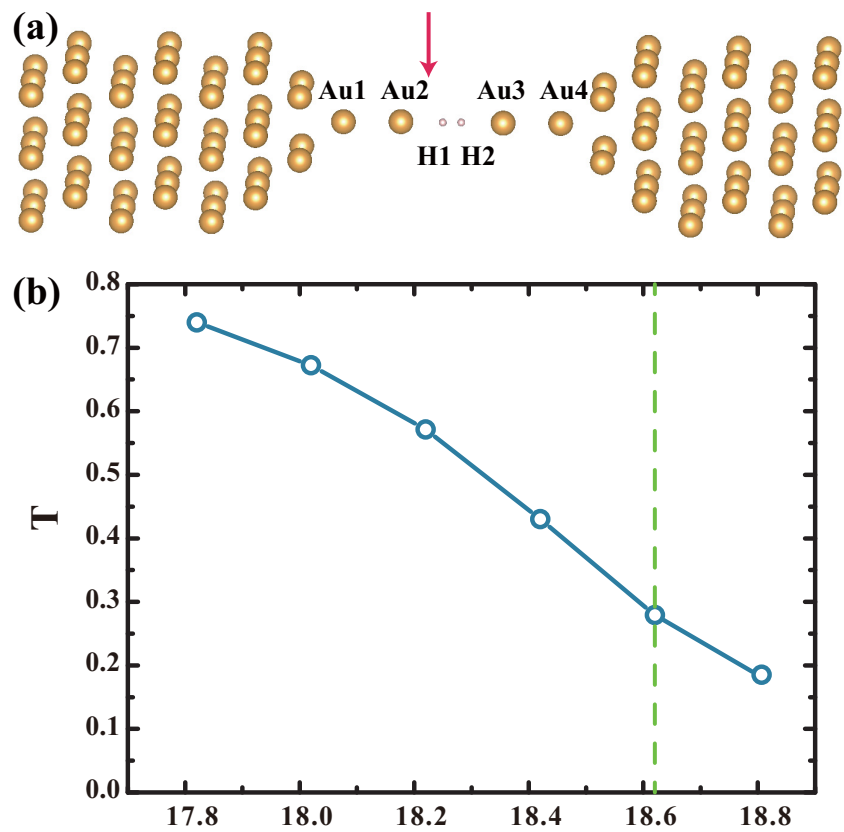

(c)
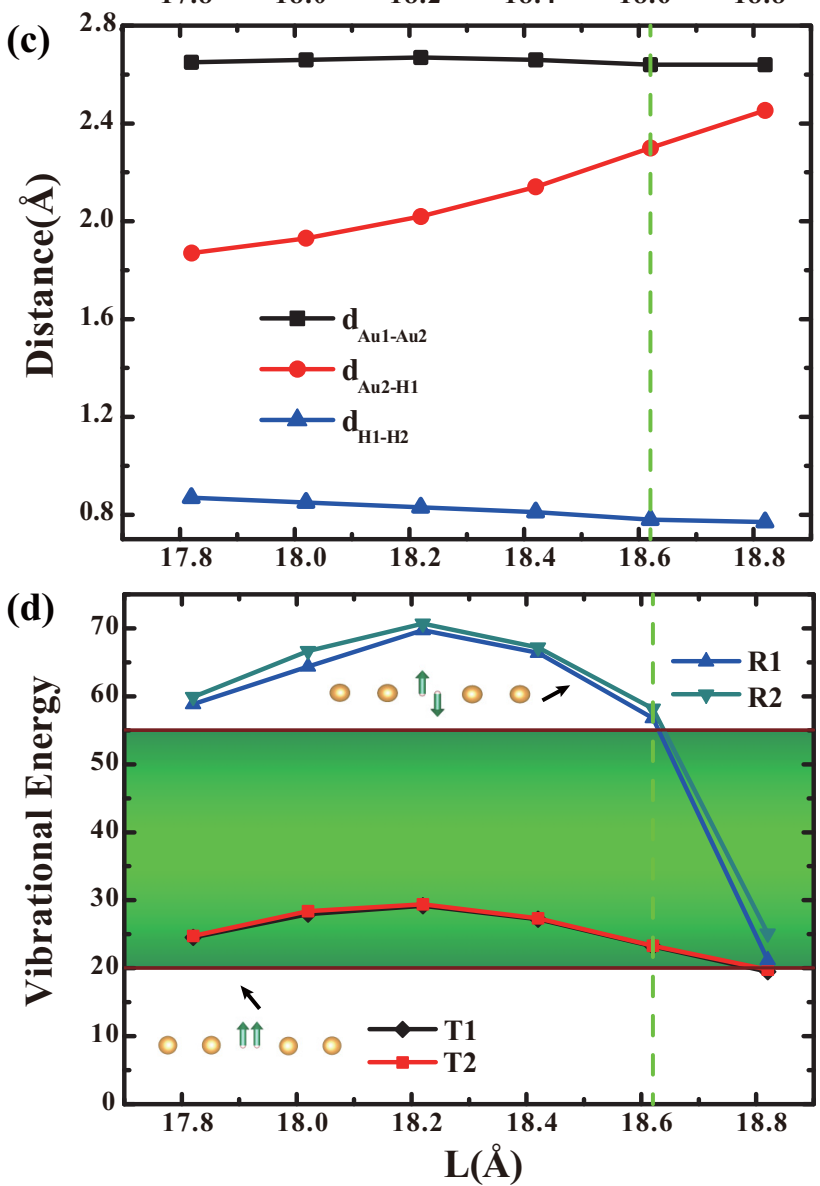

FIG. 1. (a) The optimized atomic structure of the symmetric $\mathrm{Au}-\mathrm{H}_{2}-\mathrm{Au}$ junction with (100)-oriented gold electrodes and one single $\mathrm{H}_{2}$ molecule incorporated at the center of a four-atom-long gold monatomic chain. The electrode separation is $L=18.02 \AA$ and the red arrow indicates the rupture point. The transmission coefficient at the Fermi level (b), some typical bond distances (c), and the vibrational energies of the transverse rotation and translation modes of the $\mathrm{H}_{2}$ molecule (d) as a function of the electrode separation. The green dashed line indicates the breaking electrode separation. the double-degenerate transverse rotation mode $(R)$ in which the angle between the $\mathrm{H}-\mathrm{H}$ bond and the transport direction vibrates, and the double-degenerate transverse translation mode $(T)$ in which the center of mass of the $\mathrm{H}_{2}$ molecule vibrates along the transverse directions. We calculate these vibration modes using the frozen phonon method at each stretching step. Since the vibrational energies of the longitudinal stretching and translation modes are around several hundreds of meV, the observed vibration modes of gold contacts in hydrogen environment can only be attributed to the transverse rotation and translation modes of the incorporated $\mathrm{H}_{2}$ molecule. The calculated vibration energies of the transverse rotation and translation modes are found respectively in the $60-70 \mathrm{meV}$ and 20-30 meV range, i.e., they are either higher or lower than the peak experimentally found at $33 \mathrm{meV}$. Thus, the vibrational analysis of the $\mathrm{Au}-\mathrm{H}_{2}-\mathrm{Au}$ junction does not provide a strong support to the conjecture that molecular hydrogen dominates the properties of gold contacts in a hydrogen environment. It should be pointed out that the position of the incorporated $\mathrm{H}_{2}$ molecule in the gold monatomic chain has no significant effects on the electronic transport properties and the vibration spectrum of the Au- $\mathrm{H}_{2}-\mathrm{Au}$ junction. As shown in Fig. S1 in the Supplemental Material [47], shifting the position of the $\mathrm{H}_{2}$ molecule from the center to one end of the four-atom-long gold nanowire only influences marginally the low-bias junction conductance and the vibration energies of the transverse rotation and translation modes.

Next we investigate the electronic transport and the vibrational properties of gold junctions incorporating a single hydrogen atom. As shown in Fig. 2(a), we construct a symmetric model for an $\mathrm{Au}-\mathrm{H}-\mathrm{Au}$ junction in which a single $\mathrm{H}$ atom is inserted in the center of a four-atom-long gold chain. In contrast to the case of $\mathrm{Au}-\mathrm{H}_{2}-\mathrm{Au}$ in which the low-bias conductance shows a steep drop following the increase of the electrode separation, a flat plateau with a conductance value of $0.8 G_{0}$ arises in the elongation process of the $\mathrm{Au}-\mathrm{H}-\mathrm{Au}$ junction [see Fig. 2(b)]. This originates from the strong electronic coupling between the $\mathrm{H} 1 s$ and the Au $6 s$ orbitals, resulting in a nearly perfect conducting channel around the Fermi level [20]. Due to the flexibility of the Au-H bonds, the hydrogen impurity can locate at one side of the gold monatomic chain with the $\mathrm{Au}-\mathrm{H}-\mathrm{Au}$ bond angle $\alpha=122^{\circ}$ when the gold nanowire is in a compressed state $(L=16.65 \AA)$. For larger electrode separation, the hydrogen impurity moves into the center of the two adjacent $\mathrm{Au}$ atoms with the bond angle $\alpha$ approaching $180^{\circ}$. In the chain the $\mathrm{Au}-\mathrm{H}$ bond is more robust than the $\mathrm{Au}-\mathrm{Au}$ one, and thus the $\mathrm{Au}-\mathrm{H}-\mathrm{Au}$ bond distance can stretch up to $3.76 \AA$ before the rupture of the junction, which occurs for an Au1-Au2 (Au3-Au4) separation of $3 \AA$. This is in good agreement with previous studies $[5,8]$. At variance with the previous case of molecular hydrogen, a single $\mathrm{H}$ atom inserted in the gold nanowire has only three translation modes. The two transverse translation modes vibrating in the directions perpendicular to and parallel with the plane accommodating $\mathrm{H}$ and its two adjacent $\mathrm{Au}$ atoms are respectively labeled as $\mathrm{T} 1$ and $\mathrm{T} 2$, and they become degenerate when the hydrogen atom is located along the axis of the gold chain. The vibrational energies of these two transverse translation modes are calculated to be in the $20-55 \mathrm{meV}$ range even for the rather long electrode separation before the junction rupture. 


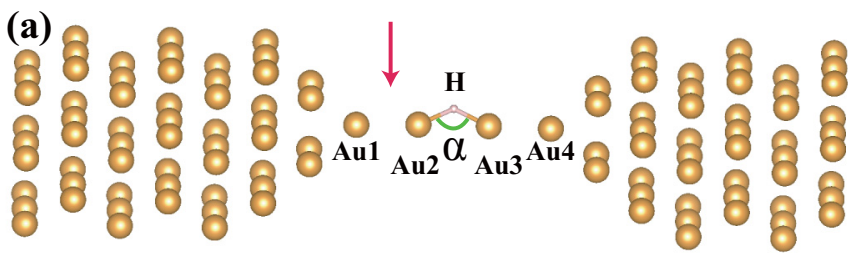

(b)
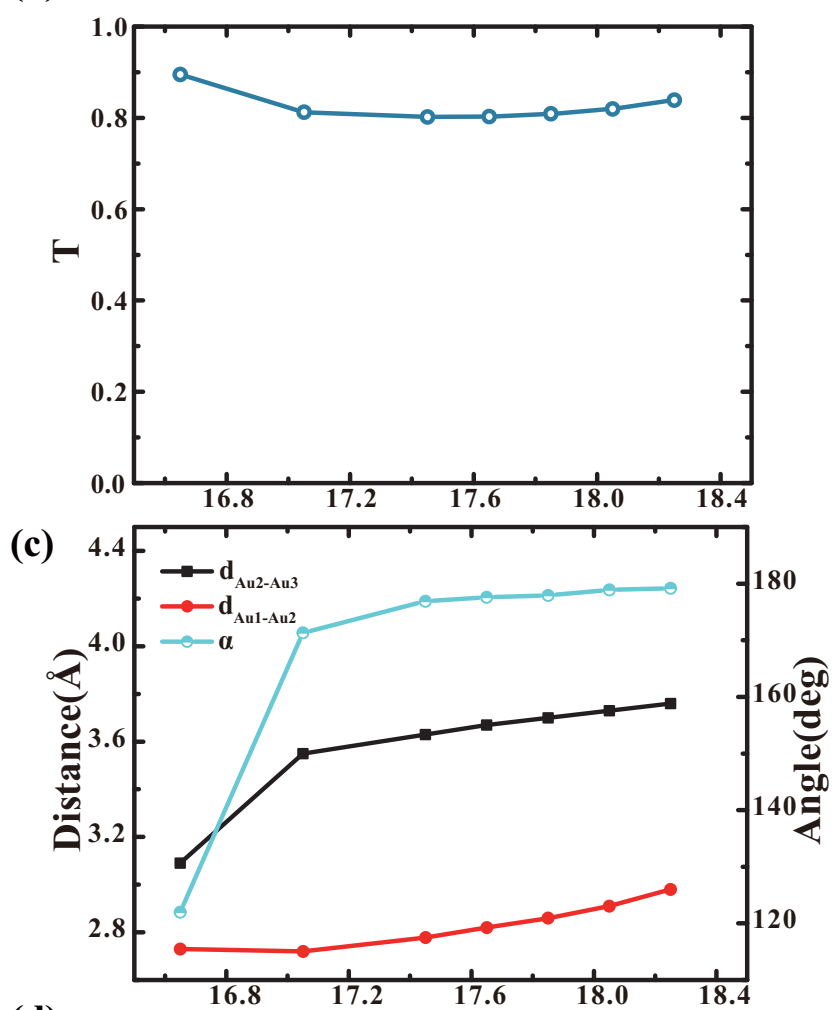

(d)

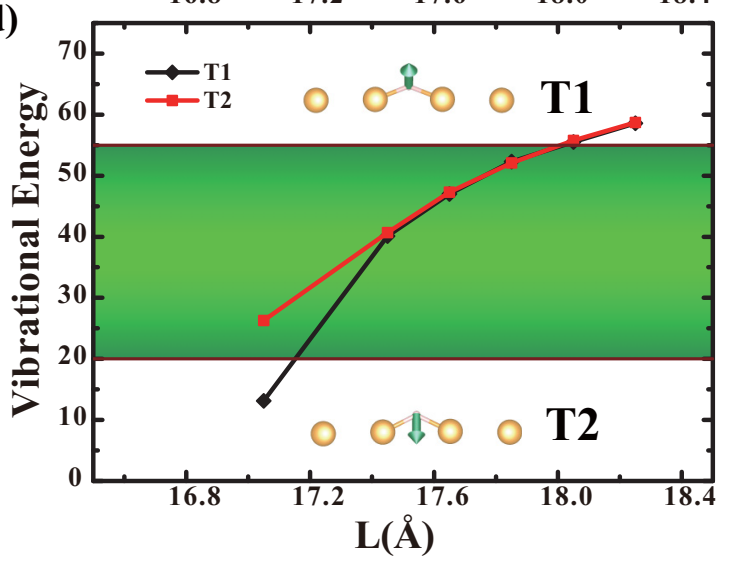

FIG. 2. (a) The optimized atomic structure of the symmetric Au$\mathrm{H}-\mathrm{Au}$ junction with (100)-oriented gold electrodes and one single $\mathrm{H}$ atom incorporated at the center of a four-atom-long gold monatomic chain. The electrode separation is $L=16.05 \AA$ and the red arrow indicates the rupture point. The transmission coefficient at the Fermi level (b), some typical bond distances and bond angle (c), and the vibrational energies of the two transverse translation modes of the $\mathrm{H}$ atom (d) as a function of the electrode separation.

Similar results are also obtained when the single $\mathrm{H}$ atom is located at one end of the four-atom-long gold monatomic chain (see Fig. S2 in the Supplemental Material) [47]. Although the distribution of the vibrational energies observed in experiments has been reproduced by the $\mathrm{Au}-\mathrm{H}-\mathrm{Au}$ junctions, the high calculated conductance values of $0.7-0.8 G_{0}$ contradict the low measured ones. Thus, gold contacts incorporating a single atomic $\mathrm{H}$ are still not appropriate to describe in full the experiments.

It has been shown that stretched gold nanowires are exceptional catalysts due to the upshift of the $d$ bands produced by the reduced coordination and the applied strain $[23,24]$. In fact, $\mathrm{H}_{2}$ molecules are predicted to dissociate over a free suspended gold nanowire with a small activation barrier of $0.1 \mathrm{eV}[19,22,25]$. Noticing that the zero-point energy of $\mathrm{H}_{2}$ molecules is $\sim 0.25 \mathrm{eV}$, it is easy to realize that $\mathrm{H}_{2}$ molecules can dissociate on a gold monatomic chain, thus forming an $\mathrm{Au}-\mathrm{H}-\mathrm{Au}-\mathrm{H}-\mathrm{Au}$ double-bridge structure. For example, Zanchet et al. have shown that $\mathrm{H}_{2}$ can dissociate into such double-bridge geometry by analyzing the minimum-energy path of a hydrogen molecule impinging on a four-atom gold wire [25]. We then investigate the electronic transport properties and the vibration spectrum of the $\mathrm{Au}-\mathrm{H}-\mathrm{Au}-\mathrm{H}-\mathrm{Au}$ double-bridge structure. Such a junction model composed of a four-atom-long gold monatomic chain and two single hydrogen atoms adsorbed at two consecutive bridge sites is shown in Fig. 3(a). The junction geometry and the corresponding low-bias conductance depend on the electrode separation. As can be seen from Figs. 3(b) and 3(c), during the initial stretching stage for electrode separations of $16.45 \AA \leqslant L<18.05 \AA$ the two distances $d_{\text {Au1-H1-Au2 }}$ and $d_{\mathrm{Au} 2-\mathrm{H} 2-\mathrm{Au} 3}$ increase constantly and the two bond angles $\angle \mathrm{Au} 1-\mathrm{H} 1-\mathrm{Au} 2$ and $\angle \mathrm{Au} 2-\mathrm{H} 2-\mathrm{Au} 3$ are enlarged from $108^{\circ}$ to $180^{\circ}$, whereas the bond length $d_{\mathrm{Au} 3-\mathrm{Au} 4}$ remains almost unchanged. As such, upon stretching the two adsorbed $\mathrm{H}$ atoms gradually move into the four-atom-long gold monatomic chain forming a coaxial structure. At the same time, the low-bias junction conductance increases from $0.14 G_{0}$ at $L=16.45 \AA$ to $0.27 G_{0}$ at $L=17.65 \AA$, showing a pronounced positive slope with the junction elongation. After the two $\mathrm{H}$ atoms and the four $\mathrm{Au}$ ones become aligned in a straight line, further stretching produces the $\mathrm{Au} 3-\mathrm{Au} 4$ bond to elongate together with the two distances $d_{\mathrm{Au} 1-\mathrm{H} 1-\mathrm{Au} 2}$ and $d_{\mathrm{Au} 2-\mathrm{H} 2-\mathrm{Au} 3}$. Finally the chain breaks at the Au3-Au4 bond [9]. During such structural modifications of the junction, the low-bias conductance shows a flat plateau near $0.26 G_{0}$, in good agreement with the measured value of $0.25 G_{0}$ [14]. Notably, the four-atom-long gold monatomic chain incorporating two single hydrogen atoms can sustain an elongation of about $2.8 \AA$. Thus, the shape, length, and the plateau value of the calculated conductance curve all reproduce well the experimental results obtained by Csonka et al. [14].

The vibrational analysis of this junction model is summarized in Fig. 3(d). Since the energies of the vibration modes related to the $\mathrm{Au}$ atoms are less than $20 \mathrm{meV}$ due to the heavier mass of gold, only the motion of the two $\mathrm{H}$ atoms can possibly provide vibrational energies larger than $20 \mathrm{meV}$. Bearing a resemblance to the $\mathrm{H}_{2}$ molecule, the dynamical properties of the two adsorbed $\mathrm{H}$ atoms can be characterized by two transverse translation modes $(T 1, T 2)$ and two transverse rotation modes $(R 1, R 2)$. In the $T 1$ and $R 1$ modes, the two hydrogen atoms vibrate in the direction perpendicular to the plane constituted by themselves and 

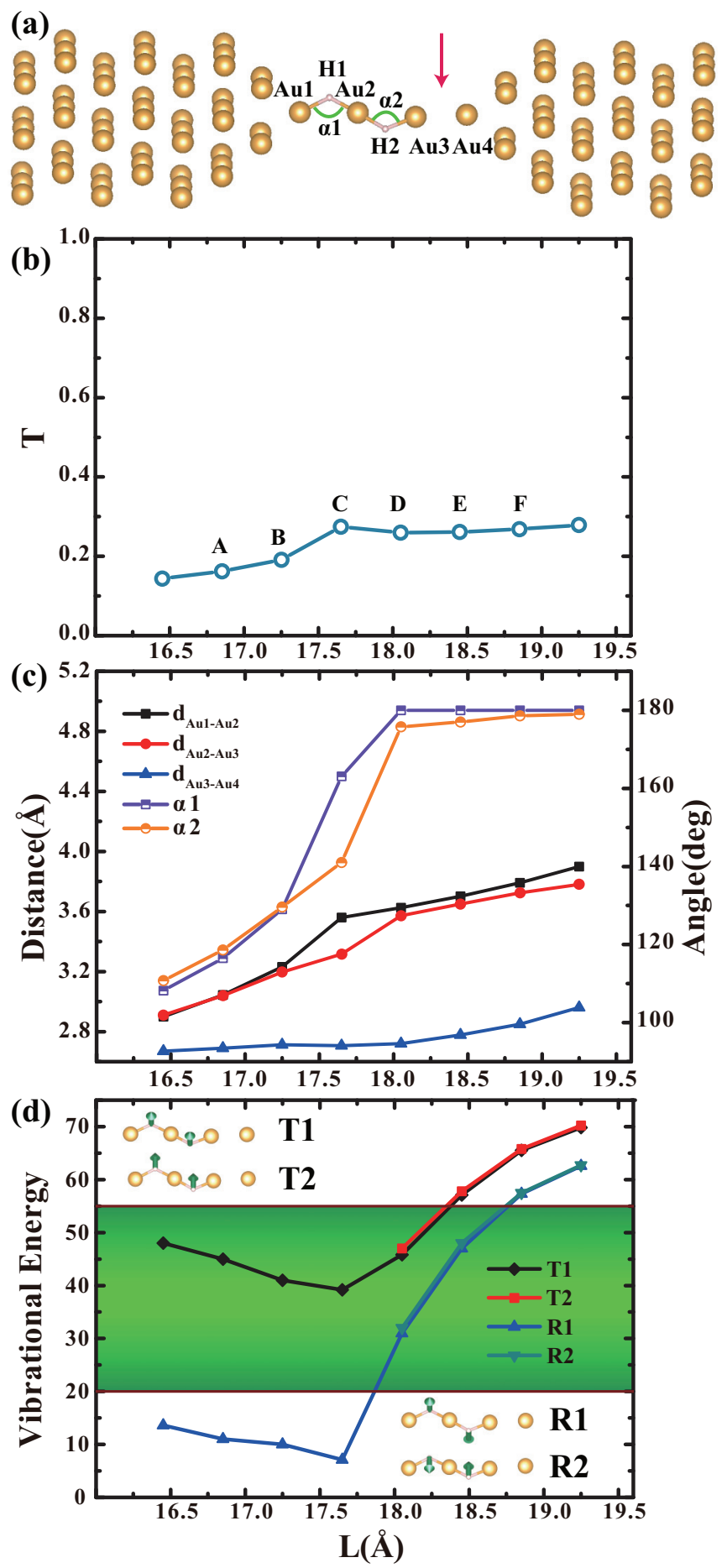

FIG. 3. (a) The optimized atomic structure of the gold nanojunction with (100)-oriented gold electrodes and the $\mathrm{Au}-\mathrm{H}-\mathrm{Au}-\mathrm{H}-\mathrm{Au}$ double-bridge structure forming on a four-atom-long gold monatomic chain. The electrode separation is $L=16.85 \AA$ and the red arrow indicates the rupture point. The transmission coefficient at the Fermi level (b), some typical bond distances and bond angles (c), and the vibrational energies of the transverse rotation and translation modes of the two $\mathrm{H}$ atoms (d) as a function of the electrode separation.

the gold chain. In contrast, in the $T 2$ and $R 2$ modes they vibrate parallel to the same plane and perpendicular to the axis of the gold chain. It should be noted that the $T 2$ and $R 2$ modes have vibrational energies around hundreds of $\mathrm{meV}$ for electrode separations $16.45 \AA \leqslant L<18.05 \AA$. We attribute this observation to the bending of the two Au-H-Au bond angles for which far more energy is needed for the hydrogen atoms to squeeze the adjacent $\mathrm{Au}$ atoms. However, the $T 1$ and $T 2$ modes as well as the $R 1$ and $R 2$ ones become degenerate as the two $\mathrm{H}$ atoms line up along the axis of the gold chain. As we can see, in most of the stretching process there is at least one vibration mode whose vibrational energy is in the 20-55 meV range, consistent with the reported experimental results [15]. Since both the calculated conductance curve and the distribution of the vibrational energies reproduce excellently the experimental results, our calculations strongly support the idea that molecular hydrogen dissociates when adsorbed on stretched gold monatomic chains. Furthermore, the Au-H-Au-H-Au double-bridge structure is the atomic arrangement that describes the experiments in a more consistent way than those with molecular or single atomic hydrogen.

It has been shown that the conductance of clean monatomic gold wires can vary significantly depending on the wire stretching and the number of incorporated atoms [48]. Therefore, in order to verify whether the number of the suspended gold atoms in the gold nanowire affects the electronic transport and vibrational properties of the double-bridge structure, we have also considered three-atom-long and five-atom-long gold monatomic chains adsorbed with two hydrogen atoms on consecutive bridge sites (Figs. S3 and S4 in the Supplemental Material) [47]. Our calculations show that both the conductance curve and the distribution of the vibration energies for these two new junctions are almost identical to those shown in Figs. 3(b) and 3(d) except that the conductance plateau is a little shorter for the three-atom-long gold chain. This weak length dependence was also observed in experiment [14]. Therefore we propose here a junction model to interpret the origin of the well-defined periodic behavior of the measured conductance curve (Fig. 4). Our model is basically similar to that given in Fig. 9 of Ref. [14]; the most significant difference is the replacement of the $\mathrm{H}_{2}$ molecule with two single hydrogen atoms. As a gold nanocontact is pulled apart, a gold monatomic chain is formed between the two gold electrodes. When one hydrogen molecule adsorbs on the three-atom-long gold chain, it dissolves into two hydrogen atoms forming the $\mathrm{Au}-\mathrm{H}-\mathrm{Au}-\mathrm{H}-\mathrm{Au}$ double-bridge structure and also reduces the junction conductance from about $1 G_{0}$ to a small value of $\sim 0.1 G_{0}$. Then, when the electrode separation further increases, the two hydrogen atoms move towards the

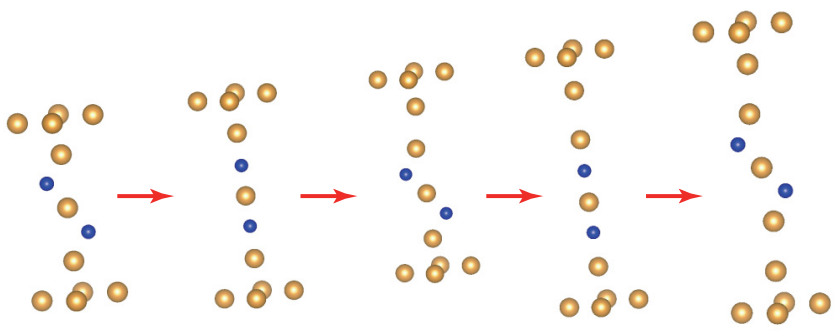

FIG. 4. The junction model proposed for the interpretation of the observed periodic structure with positive slope in the conductance curves of gold nanocontacts in hydrogen environment. 

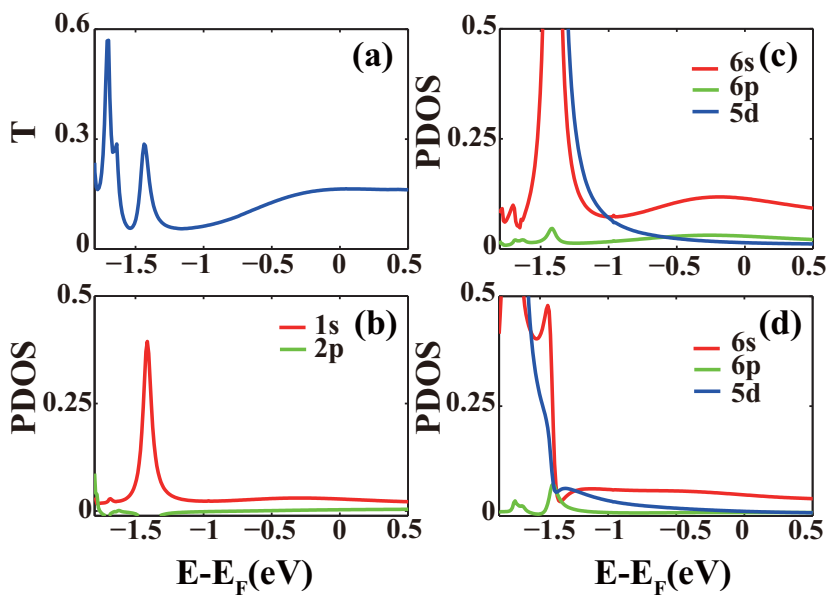

FIG. 5. The equilibrium transmission spectrum (a) and the LDOS projected onto the $1 s$ atomic orbital of the $\mathrm{H} 2$ atom (b), $6 s, 6 p$, and $5 d$ atomic orbitals of the Au3 atom (c), and the Au1 atom (d) in the junction model shown in Fig. 3(a) at the electrode separation $L=16.85 \AA$.

axis of the gold chain and the junction conductance gets larger. After these two $\mathrm{H}$ atoms align along the chain axis, the conductance curve exhibits a plateau. Due to the strong $\mathrm{Au}-\mathrm{H}$ bonding, more gold atoms can be pulled into the chain during the stretching process. Once a new gold atom from the electrode is added into the chain, the two hydrogen atoms go back to the bridge sites with smaller conductance and a new cycle starts again until the junction finally ruptures (see Fig. 4).

A deep understanding of the particular conductance curve of the double-bridge structures can be obtained by comparing the transmission spectra and the local density of states (LDOS) projected onto the atomic orbitals of the hydrogen and gold atoms in the constriction. Figure 5(a) presents the equilibrium transmission spectrum of the junction model shown in Fig. 3(a) at the electrode separation of $L=16.85 \AA$. A noticeable transmission peak appears at $1.43 \mathrm{eV}$ below $E_{\mathrm{F}}$ with its tail extending above $E_{\mathrm{F}}$. By comparing the LDOS of the Au1, H1, $\mathrm{Au} 2, \mathrm{H} 2$, and $\mathrm{Au} 3$ atoms in the junction, we can find that the transmission around $E_{\mathrm{F}}$ is mainly due to the $\mathrm{H} 1 s$ and $\mathrm{Au} 6 s$ atomic orbitals. This is also corroborated by the eigenchannel analysis $[49,50]$, which clearly shows that around the Fermi level there is only one conducting channel dominated by the $\mathrm{H} 1 s$ and $\mathrm{Au} 6 s$ atomic orbitals (Fig. S5 in the Supplemental Material) [47]. As the junction is stretched, this transmission peak moves to higher energies; the transmission coefficient at $E_{\mathrm{F}}$ increases at the initial stages and then remains almost constant (Fig. 6). This is a cooperative result determined by the complex interaction among the hydrogen and gold atoms in the constriction. When the two $\mathrm{H}$ atoms are located at the bridge sites, the shape of the transmission spectra around $E_{\mathrm{F}}$ is more similar to that of the LDOS of the Au3 $6 s$ atomic orbital; in contrast, after the two $\mathrm{H}$ atoms enter the gold chain the shape of the transmission spectra is more similar to that of the LDOS of the Au1 $6 s$ atomic orbital. Therefore, the occurrence of the well-defined periodic structure with a pronounced positive slope in the low-bias conductance region
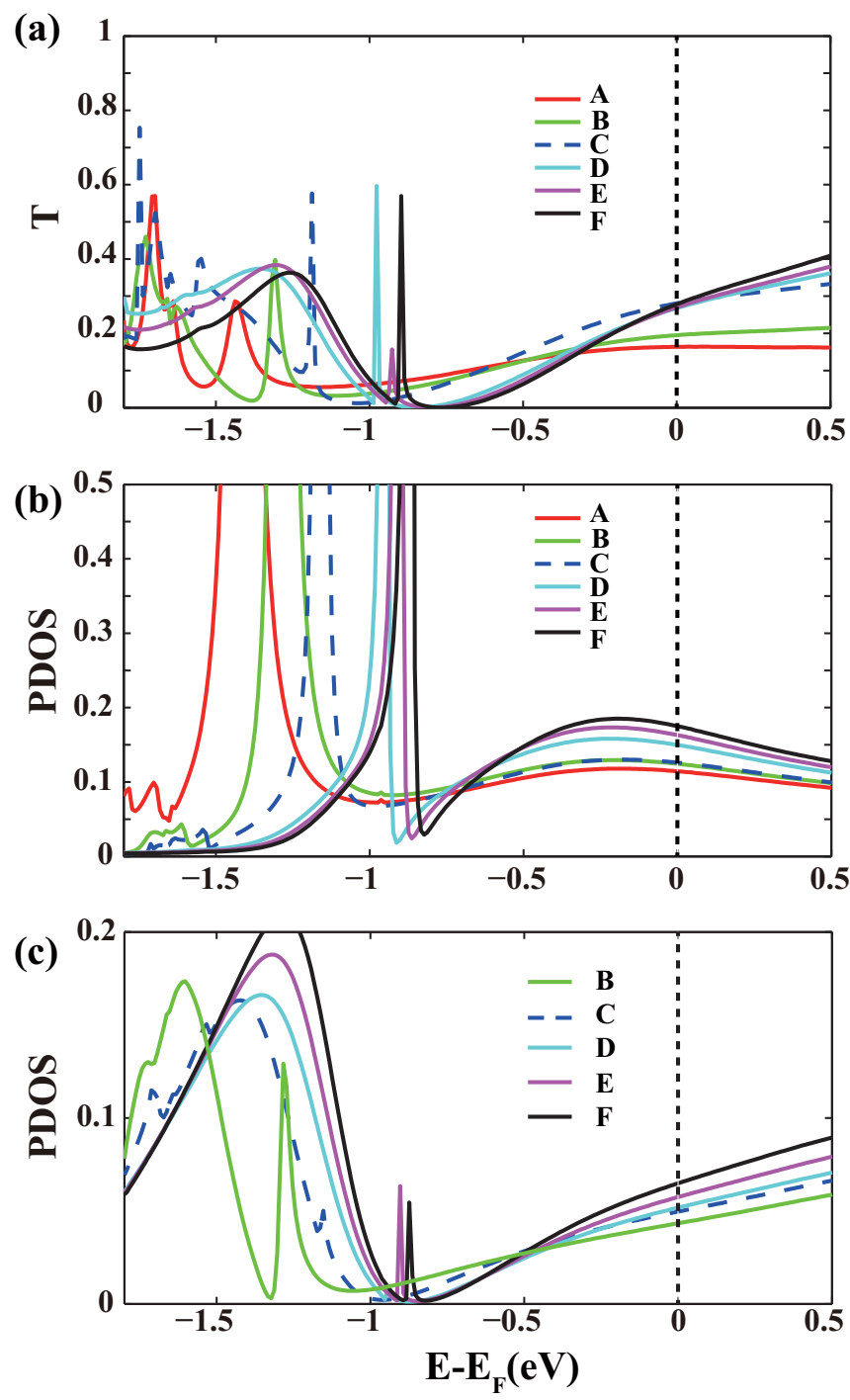

FIG. 6. The equilibrium transmission spectra (a) and the LDOS projected onto the $6 s$ atomic orbitals of the Au3 atom (b) and the $\mathrm{Au} 1$ atom (c) in the junction model shown in Fig. 3(a) at the different electrode separations, which are labeled in Fig. 3(b).

should be ascribed to the specific electronic interactions in the double-bridge structures.

It has been shown that the electrode orientation significantly affects the calculated transport properties of molecular junctions [51,52]. Therefore, we have also investigated the low-bias conductance and the vibration spectrum of the $\mathrm{Au}-\mathrm{H}-\mathrm{Au}-\mathrm{H}-\mathrm{Au}$ double-bridge structure connected to two (111)-oriented Au electrodes (Fig. 7). Similarly to the cases of the $\mathrm{Au}(100)$ electrodes, as the junction is stretched the low-bias conductance increases initially and then remains at $0.23 G_{0}$, further supporting the conclusion that the experimentally observed periodic behavior of the conductance curve of gold contacts in hydrogen environment can be assigned to the gold monatomic chains adsorbed with two hydrogen atoms forming the double-bridge structure. Some of the calculated vibrational energies are also in the $20-55 \mathrm{meV}$ range, showing a very weak dependence on the electrode orientation. 

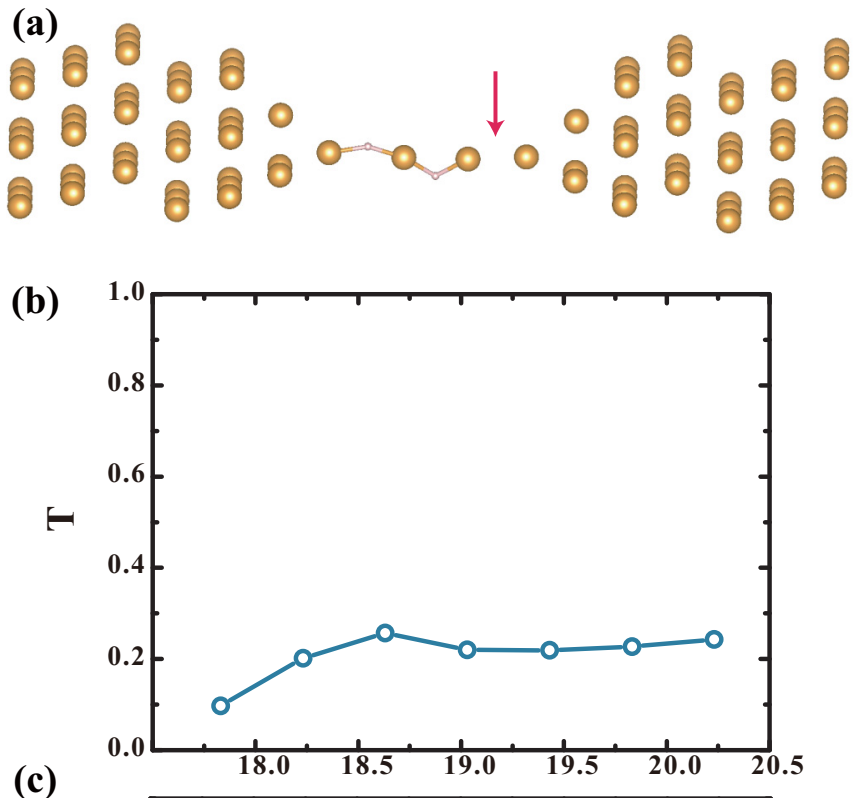

(c)

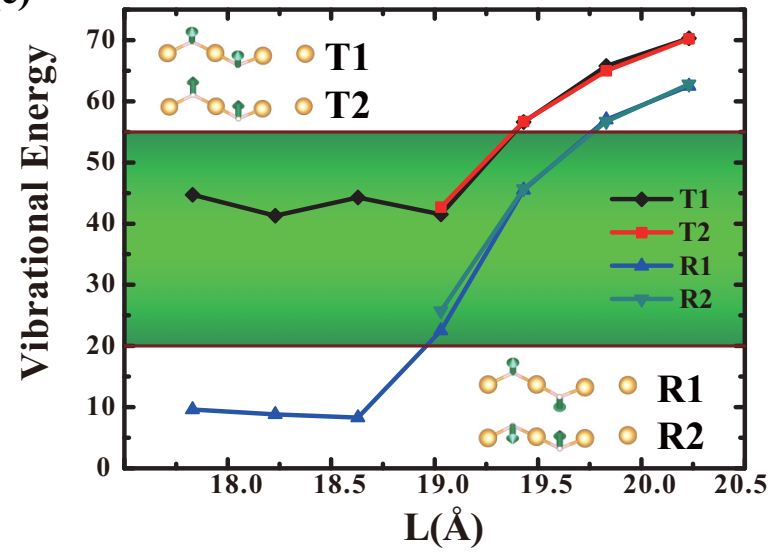

FIG. 7. (a) The optimized atomic structure of the gold nanojunction with the (111)-oriented gold electrodes and the $\mathrm{Au}-\mathrm{H}-\mathrm{Au}-\mathrm{H}-\mathrm{Au}$ double-bridge structure forming on a four-atom-long gold monatomic chain, the electrode separation $L=18.23 \AA$ and the red arrow indicating the rupture point; the transmission coefficient at the Fermi level (b) and the vibrational energies of the transverse rotation and translation modes of the two $\mathrm{H}$ atoms (c) as a function of the electrode separation.

\section{CONCLUSION}

In this study we have investigated the atomic structure, vibrational and electronic transport properties of gold atomic junctions doped with molecular and atomic hydrogen employing the NEGF+DFT approach. Our calculations show that gold nanocontacts incorporating a single $\mathrm{H}_{2}$ molecule or a single $\mathrm{H}$ atom cannot produce conductance curves consistent with those observed in experiments for gold atomic junctions in hydrogen environment. In contrast, when one $\mathrm{H}_{2}$ molecule adsorbed on a stretched gold monatomic chain dissociates into two $\mathrm{H}$ atoms and forms the $\mathrm{Au}-\mathrm{H}-\mathrm{Au}-\mathrm{H}-\mathrm{Au}$ double-bridge structure, the calculated conductance curve presents a pronounced increase in the initial stretching process and then exhibits a plateau with a conductance of $0.26 G_{0}$ until the rupture of the chain. Since this specific conductance behavior has been obtained for gold junctions with gold monatomic chains composed of three, four, and five atoms, we can construct a junction model with the $\mathrm{Au}-\mathrm{H}-\mathrm{Au}-\mathrm{H}-\mathrm{Au}$ double-bridge structure formed on length-varied gold monatomic chains to interpret the observed periodic structure with positive slope in the low-conductance region. Furthermore, one or more of the transverse translation and rotation modes of the two $\mathrm{H}$ atoms in the $\mathrm{Au}-\mathrm{H}-\mathrm{Au}-\mathrm{H}-\mathrm{Au}$ double-bridge structure have vibration energies in the $20-55 \mathrm{meV}$ range, in good agreement with the experimental values. Therefore, the $\mathrm{Au}-\mathrm{H}-\mathrm{Au}-\mathrm{H}-\mathrm{Au}$ doublebridge structure is the more preferred atomic configuration for gold junctions formed in hydrogen environment. These findings not only provide an explanation for the observed vibrational and electronic transport properties of gold atomic junctions in hydrogen environment but also demonstrate the applications of gold monatomic chains as chemical sensors and catalysts.

\section{ACKNOWLEDGMENTS}

This project was supported by the National Natural Science Foundation of China (Grants No. 61321001 and No. 61271050) and the MOST of China (Grant No. 2013CB933404). S.S. acknowledges additional funding support from the European Research Council (QUEST project) and by AMBER (12/RC/2278).
[1] N. Agraï, A. L. Yeyati, and J. M. van Ruitenbeek, Phys. Rep. 377, 81 (2003).

[2] B. Hammer and J. K. Nørskov, Nature (London) 376, 238 (1995).

[3] S. R. Bahn, N. Lopez, J. K. Nørskov, and K. W. Jacobsen, Phys. Rev. B 66, 081405(R) (2002).

[4] N. V. Skorodumova and S. I. Simak, Phys. Rev. B 67, 121404(R) (2003).

[5] F. D. Novaes, A. J. R. da Silva, E. Z. da Silva, and A. Fazzio, Phys. Rev. Lett. 90, 036101 (2003).

[6] E. Hobi, Jr., A. J. R. da Silva, F. D. Novaes, E. Z. da Silva, and A. Fazzio, Phys. Rev. Lett. 95, 169601 (2005).

[7] E. Hobi, Jr., A. Fazzio, and A. J. R. da Silva, Phys. Rev. Lett. 100, 056104 (2008).
[8] F. D. Novaes, E. Z. da Silva, A. J. R. da Silva, and A. Fazzio, Surf. Sci. 566-568, 367 (2004).

[9] E. Z. da Silva, F. D. Novaes, A. J. R. da Silva, and A. Fazzio, Nanoscale Res. Lett. 1, 91 (2006).

[10] N. V. Skorodumova, S. I. Simak, A. E. Kochetov, and B. Johansson, Phys. Rev. B 75, 235440 (2007).

[11] P. Vélez, S. A. Dassie, and E. P. M. Leiva, Phys. Rev. B 81, 125440 (2010).

[12] T. Kizuka, Phys. Rev. B 77, 155401 (2008).

[13] S. Csonka, A. Halbritter, G. Mihály, E. Jurdik, O. I. Shklyarevskii, S. Speller, and H. van Kempen, Phys. Rev. Lett. 90, 116803 (2003).

[14] S. Csonka, A. Halbritter, and G. Mihály, Phys. Rev. B 73, 075405 (2006). 
[15] M. Kiguchi, T. Nakazumi, K. Hashimoto, and K. Murakoshi, Phys. Rev. B 81, 045420 (2010).

[16] T. Nakazumi, S. Kaneko, and M. Kiguchi, J. Phys. Chem. C 118, 7489 (2014).

[17] Y. Li, S. Kaneko, S. Fujii, and M. Kiguchi, J. Phys. Chem. C 119, 19143 (2015).

[18] R. N. Barnett, H. Häkkinen, A. G. Scherbakov, and U. Landman, Nano Lett. 4, 1845 (2004).

[19] P. Jelínek, R. Pérez, J. Ortega, and F. Flores, Phys. Rev. Lett. 96, 046803 (2006).

[20] T. Frederiksen, M. Paulsson, and M. Brandbyge, J. Phys.: Conf. Ser. 61, 312 (2007).

[21] P. Jelínek, R. Pérez, J. Ortega, and F. Flores, Phys. Rev. B 77, 115447 (2008).

[22] A. Zanchet, A. Dorta-Urra, O. Roncero, F. Flores, C. Tablero, M. Paniagua, and A. Aguado, Phys. Chem. Chem. Phys. 11, 10122 (2009).

[23] E. Santos, P. Quaino, G. Soldano, and W. Schmickler, Electrochem. Commun. 11, 1764 (2009).

[24] D. Cakir and O. Gulseren, Phys. Rev. B 84, 085450 (2011).

[25] A. Zanchet, O. Roncero, A. Dorta-Urra, A. Aguado, J. I. Martínez, F. Flores, and N. Lorente, Phys. Rev. B 90, 041404(R) (2014).

[26] H. Ohnishi, Y. Kondo, and K. Takayanagi, Nature (London) 395 , 780 (1998).

[27] Y. Kondo and K. Takayanagi, Science 289, 606 (2000).

[28] V. Rodrigues, T. Fuhrer, and D. Ugarte, Phys. Rev. Lett. 85, 4124 (2000).

[29] V. Rodrigues and D. Ugarte, Phys. Rev. B 63, 073405 (2001).

[30] S. B. Legoas, D. S. Galvão, V. Rodrigues, and D. Ugarte, Phys. Rev. Lett. 88, 076105 (2002).

[31] Y. Meir and N. S. Wingreen, Phys. Rev. Lett. 68, 2512 (1992).

[32] P. Hohenberg and W. Kohn, Phys. Rev. 136, B864 (1964).

[33] W. Kohn and L. J. Sham, Phys. Rev. 140, A1133 (1965).

[34] Y. Xue, S. Datta, and M.A. Ratner, Chem. Phys. 281, 151 (2002).

[35] M. Brandbyge, J.-L. Mozos, P. Ordejón, J. Taylor, and K. Stokbro, Phys. Rev. B 65, 165401 (2002).

[36] J. Zhang, S. Hou, R. Li, Z. Qian, R. Han, Z. Shen, X. Zhao, and Z. Xue, Nanotechnology 16, 3057 (2005).
[37] R. Li, J. Zhang, S. Hou, Z. Qian, Z. Shen, X. Zhao, and Z. Xue, Chem. Phys. 336, 127 (2007).

[38] A. R. Rocha, V. M. García-Suárez, S. W. Bailey, C. J. Lambert, J. Ferrer, and S. Sanvito, Nat. Mater. 4, 335 (2005).

[39] A. R. Rocha, V. M. García-Suárez, S. Bailey, C. Lambert, J. Ferrer, and S. Sanvito, Phys. Rev. B 73, 085414 (2006).

[40] I. Rungger and S. Sanvito, Phys. Rev. B 78, 035407 (2008).

[41] J. M. Soler, E. Artacho, J. D. Gale, A. García, J. Junquera, P. Ordejón, and D. Sánchez-Portal, J. Phys.: Condens. Matter 14, 2745 (2002).

[42] N. Troullier and J. L. Martins, Phys. Rev. B 43, 1993 (1991).

[43] J. P. Perdew, K. Burke, and M. Ernzerhof, Phys. Rev. Lett. 77, 3865 (1996).

[44] C. Kittel, Introduction to Solid State Physics, 8th ed. (Wiley, Hoboken, NJ, 2004).

[45] P. C. H. Mitchell, P. Wolohan, D. Thompsett, and S. J. Cooper, J. Mol. Catal. A: Chem. 119, 223 (1997).

[46] D. A. Mcquarrie and J. D. Simon, Physical Chemistry: A Molecular Approach (University Science Books, Sausalito, CA, 1997).

[47] See Supplemental Material at http://link.aps.org/supplemental/ 10.1103/PhysRevB.93.125438 for the atomic structure, transport property, and vibration energies of the asymmetric Au$\mathrm{H}_{2}-\mathrm{Au}$ and $\mathrm{Au}-\mathrm{H}-\mathrm{Au}$ junctions, the gold nanocontacts with the $\mathrm{Au}-\mathrm{H}-\mathrm{Au}-\mathrm{H}-\mathrm{Au}$ double-bridge structure forming on threeatom-long and five-atom-long gold chains, and the eigenchannel analysis of the gold nanocontact with the $\mathrm{Au}-\mathrm{H}-\mathrm{Au}-\mathrm{H}-\mathrm{Au}$ double-bridge structure forming on a four-atom-long gold chain.

[48] A. Grigoriev, N. V. Skorodumova, S. I. Simak, G. Wendin, B. Johansson, and R. Ahuja, Phys. Rev. Lett. 97, 236807 (2006).

[49] R. Li, S. Hou, J. Zhang, Z. Qian, Z. Shen, and X. Zhao, J. Chem. Phys. 125, 194113 (2006).

[50] M. Paulsson and M. Brandbyge, Phys. Rev. B 76, 115117 (2007).

[51] S.-H. Ke, H. U. Baranger, and W. Yang, J. Chem. Phys. 123, 114701 (2005).

[52] X. Tu, M. Wang, S. Sanvito, and S. Hou, J. Chem. Phys. 141, 194702 (2014). 